\title{
THE
}

UNIVERSITY

University of Rhode Island

OF RHODE ISLAND

DigitalCommons@URI

12-12-2016

\section{Solution-Based Photo-Patterned Gold Film Formation on Silicon Nitride}

\author{
Y.M. Nuwan D.Y. Bandara \\ University of Rhode Island \\ Buddini Iroshika Karawdeniya \\ University of Rhode Island \\ Julie C. Whelan \\ University of Rhode Island \\ Lucas D.S. Ginsberg \\ University of Rhode Island

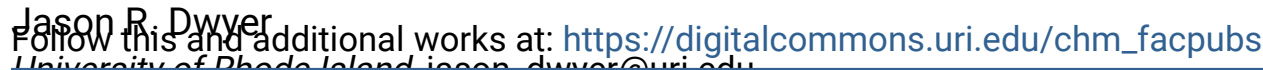 \\ The University of Rhode Island Faculty have made this article openly available. \\ Please let us know how Open Access to this research benefits you.
}

This is a pre-publication author manuscript of the final, published article.

Terms of Use

This article is made available under the terms and conditions applicable towards Open Access

Policy Articles, as set forth in our Terms of Use.

\section{Citation/Publisher Attribution}

Bandara, Y. M. Nuwan D. Y., Karawdeniya, B. I., Whelan, J. C., Ginsberg, L. D.S., \& Dwyer, J. R. (2016).

Solution-Based Photo-Patterned Gold Film Formation on Silicon Nitride. ACS Appl. Mater. Interfaces, 8(51), 34964-34969. doi: 10.1021/acsami.6b12720

Available at: http://dx.doi.org/10.1021/acsami.6b12720

This Article is brought to you for free and open access by the Chemistry at DigitalCommons@URI. It has been accepted for inclusion in Chemistry Faculty Publications by an authorized administrator of DigitalCommons@URI. For more information, please contact digitalcommons-group@uri.edu. 


\section{Solution-Based Photo-Patterned Gold Film}

\section{Formation on Silicon Nitride.}

Y.M. Nuwan D.Y. Bandara, Buddini Iroshika Karawdeniya, Julie C. Whelan, Lucas D.S. Ginsberg ${ }^{\S}$, and Jason R. Dwyer*.

Final version of manuscript available at

https://pubs.acs.org/doi/abs/10.1021/acsami.6b12720 (Supporting information available for free at https://pubs.acs.org/doi/suppl/10.1021/acsami.6b12720)

ACS Appl. Mater. Interfaces, 2016, 8 (51), pp 34964-34969

DOI: 10.1021 acsami.6b12720 


\title{
Solution-Based Photo-Patterned Gold Film
}

\section{Formation on Silicon Nitride.}

\author{
Y.M. Nuwan D.Y. Bandara, Buddini Iroshika Karawdeniya, Julie C. Whelan, Lucas D.S.
}

Ginsberg§, and Jason R. Dwyer*.

Department of Chemistry, University of Rhode Island, 140 Flagg Road, Kingston, RI, 02881, United States.

ABSTRACT: Silicon nitride fabricated by low-pressure chemical vapor deposition (LPCVD) to be silicon-rich $\left(\mathrm{SiN}_{\mathrm{x}}\right)$, is a ubiquitous insulating thin film in the microelectronics industry, and an exceptional structural material for nanofabrication. Free-standing $<100 \mathrm{~nm}$-thick $\operatorname{SiN}_{\mathrm{x}}$ membranes are especially compelling, particularly when used to deliver forefront molecular sensing capabilities in nanofluidic devices. We developed an accessible, gentle, and solution-based photodirected surface metallization approach well-suited to forming patterned metal films as integral structural and functional features in thin-membrane-based $\mathrm{SiN}_{\mathrm{x}}$ devices-for use as electrodes or surface chemical functionalization platforms, for example-augmenting existing device capabilities and properties for a wide range of applications.

KEYWORDS. Patterned metallization; Photocontrolled metallization; Silicon nitride covalent photomasking; Silicon nitride surface functionalization; Silicon nitride membrane; Thin gold films; Electroless plating; Hydrosilylation. 
Thin, silicon-rich silicon nitride films prepared by low pressure chemical vapor deposition $\left(\operatorname{LPCVD~SiN}_{\mathrm{x}}\right)$ are a prevalent element of micro- and nanofabricated devices and they can be used to confer mechanical and chemical robustness, diffusion inhibition, and dielectric strength. ${ }^{1-3}$ Devices and applications exploiting these beneficial native features can be augmented and improved using designer metal overlayers that fulfill structural roles, serve as electrodes, and provide alternative surface chemistry options, including as a platform for subsequent thiol monolayer self-assembly. The field of nanopore single-molecule sensing offers compelling examples of the prospects of merging $\mathrm{SiN}_{\mathrm{x}}$ thin films and designer metal layers into devices, and does this within a nanofluidic context where the need for versatile metallizing approaches is clear. ${ }^{3-}$ ${ }^{7}$ The most common solid-state nanopores are $<100 \mathrm{~nm}$-diameter nanofluidic channels formed through $<100 \mathrm{~nm}$-thick, free-standing $\mathrm{SiN}_{\mathrm{x}}$ films, and nanopore-integrated metal films can enhance sensing capabilities by serving as optical elements such as light shields and plasmonic films, as electrodes for tunneling and other molecular control and sensing functions, and as a means to tune nanopore size and surface chemistry. ${ }^{3-8}$ The nanoscale dimensions of the $\mathrm{SiN}_{\mathrm{x}}$ film and pore can be significant barriers to efforts to incorporate such functional metal films, particularly when the interior of the pore must be metallized. Solution-based metallization routes offer an appealing route with natural compatibility with micro- and nanofluidic devices. In this domain, nanopores represent an extreme fabrication challenge owing to their small size in all directions. In fluidic devices more amenable to conventional micro- and nanofabrication routes, such as fluid cells for transmission electron microscopy on liquids, ${ }^{1,3}$ the channel dimensions can be nanoscale in height but millimeters in width. In such platforms, the design of low-overhead patterned metallization strategies should carefully account for the potential challenges of the $<100 \mathrm{~nm}$ thickness of the free-standing, supporting $\operatorname{SiN}_{\mathrm{x}}$ channel floor and ceiling. Surface capture of 
nanoparticles_-by specific and nonspecific attachment mechanisms — is a possible solution-based route to surface metallization. ${ }^{9-12}$ Electroless plating is a compelling alternative: a solution-based process useful for metallizing a wide variety of materials, including nonconductive and irregularlyshaped materials. ${ }^{7,13-14}$ Solution access, rather than line-of-sight as in physical vapor deposition, dictates where surface plating will occur, so that electroless plating is an appealing choice for fashioning nanofluidic devices where even irregular and concealed surfaces may require metallization. Electroless plating is a flexible fabrication technique that can metallize the interior surfaces of existing nanoconfined volumes, and also create nanoscale miniatures by conformal metal deposition inside initially larger features. ${ }^{7,14-15}$ To fully exploit solution-based metallization as a tool for micro- and nanofabrication, however, requires control not just over the plated film composition, thickness, and grain size, but also over its spatial disposition, which must be at least partly independent of underlying substrate patterning. ${ }^{15}$ We wanted a patterning approach that did not need mechanical access to target surfaces, both to improve the generality of the approach, and to minimize the risk of damage that can accompany repeated handling of thin films - especially of free-standing thin-films. We sought to develop a gentle, solution-based patterned metallization approach $^{16-17}$ capable of plating a range of even structured substrates, including inside existing (nano)fluidic channels. ${ }^{3,}$ 7, 14-15, 18 The horizons of single-molecule science have recently been dramatically expanded by the development of simple methods for fabricating nanopores: entirely solution-based processes requiring only uncomplicated instrumentation are removing barriers to the widespread use of nanopore methods. ${ }^{19}$ To conserve the benefits of simple pore formation methods, our focus also included developing similarly widely-accessible, straightfoward solutionbased approaches to patterned metallization. We therefore wanted to avoid the instrumentation and processing overhead associated with traditional photoresist-based approaches and more exotic 
analogues and alternatives. ${ }^{11,20-23}$ In the conventional approach to substrate patterning by optical lithography, the target surface is coated with photoresist, usually by spin-coating. After baking, the photoresist is irradiated through a mask, developed, and then selectively removed by dissolution or etching to reveal the patterned surface. To simplify the processing, we chose instead to only attach the protective layer where it was desired, by photo-patterning the covalent attachment of an organic monolayer to $\mathrm{SiN}_{\mathrm{x}}{ }^{24}$ and to investigate its ability to then template the substrate metallization. With the use of substrate immersion and an initially liquid patterning precursor (here, 1-octene), we sought to gain greater tolerance to irregularities-including the presence of engineered structures such as micro- and nanofluidic channels — of the $\mathrm{SiN}_{\mathrm{x}}$ surface. For metallization, we initially adopted an electroless plating approach that had been specifically developed for gold-plating $\operatorname{SiN}_{\mathrm{x} .}{ }^{7,25}$

The approach is outlined in Scheme 1, and full details of materials, instrumentation, and safety precautions are provided in the Supporting Information (SI). We had previously developed a gold electroless plating approach for $\mathrm{SiN}_{\mathrm{x}}$ that required a hydrofluoric acid (HF) etching step prior to surface metallization ${ }^{7}, 25$. The HF-etched surface offered a natural starting point to incorporate patterned monolayer formation in an effort to guide the spatial extent of the substrate metallization. An alkane monolayer can be covalently linked to a freshly HF-etched $\mathrm{SiN}_{\mathrm{x}}$ surface through the photochemically-driven hydrosilylation of a 1 -alkene. ${ }^{24}$ Tremendous care must be exercised in the use of HF, and we detail the precautions - including additional protective equipment and monitored work-in the SI. The UV (254 nm) photoirradiation was through copper transmission electron microscopy (TEM) grid masks, with different bar sizes and spacings (see SI for specifications), that had been placed directly on the wafer (without securing them or preventing liquid access underneath), with both wafer and mask then immersed in the 1-alkene. Plating 
selectivity depended on rigid adherence to the rinsing steps detailed in the SI, and, as in prior work, we ensured compatibility of the process with free-standing ultrathin $\mathrm{SiN}_{\mathrm{x}}$ membranes by avoiding ultrasonic cleaning steps. ${ }^{20}$

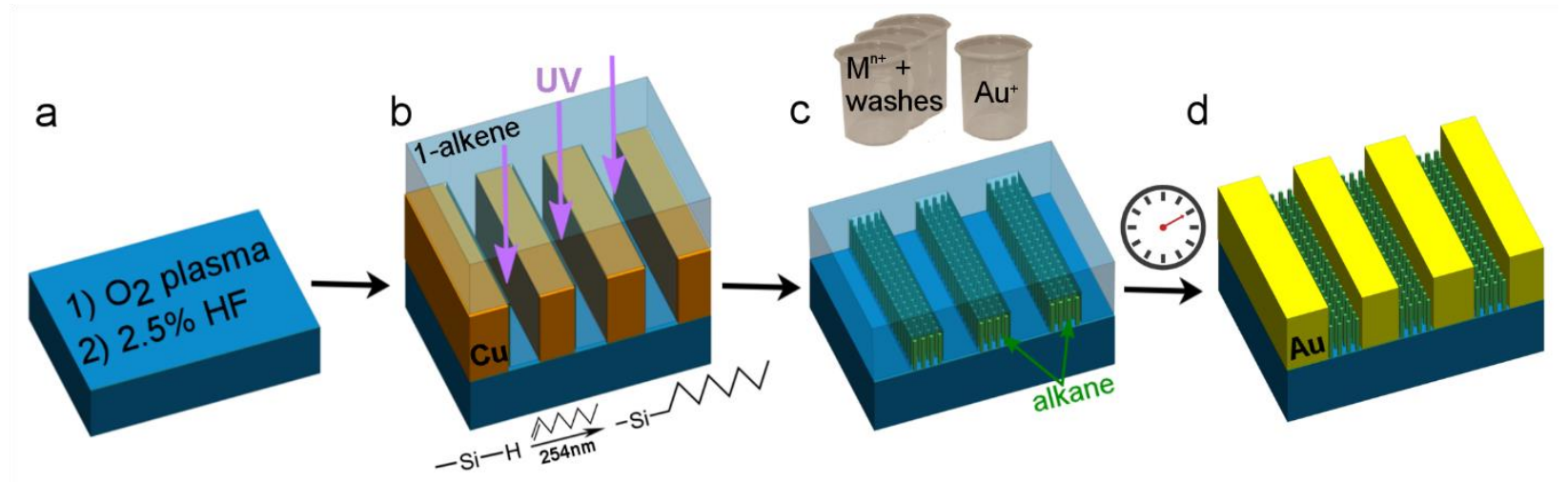

Scheme 1: A $\operatorname{SiN}_{x}$ substrate is (a) plasma treated and hydrofluoric-acid etched, then (b) immersed in 1-octene for photopatterning $(254 \mathrm{~nm})$ through a TEM grid. The patterned substrate is then (c) immersed in a series of metallizing solutions to yield (d) a patterned gold film. Structural features are not to scale. A detailed description of solution compositions and process flow is provided in the SI.

We proposed to spatially pattern LPCVD $\mathrm{SiN}_{\mathrm{x}}$ metallization by forming a physical barrier on the surface to control where the metal plating could take place. The first step of patterned plating thus involved the formation of this patterned protective layer. In our prior work to develop an electroless gold plating procedure for $\mathrm{SiN}_{\mathrm{x}}$, we found it was essential to first etch the $\mathrm{SiN}_{\mathrm{x}}$ surface with dilute HF. ${ }^{7}$ This same initial etching step forms the starting point for the covalent attachment of 1-alkenes (or 1-alkynes) by photochemical (or thermal) hydrosilylation on silicon-rich $\mathrm{SiN}_{\mathrm{x}}{ }^{2,24}$ to form alkane monolayers that could potentially function as a barriers for electroless plating. Photoirradiation using a UV lamp $(254 \mathrm{~nm})$ proved convenient in transferring the spatial patterning offered by a selection of copper transmission electron microscopy (TEM) grids (Figure 1a) to the $\mathrm{SiN}_{\mathrm{x}}$ surface. Figure $1 \mathrm{~b}$ is a photograph of a representative substrate after patterned irradiation through a thin $(<2 \mathrm{~mm})$ layer of neat 1-octene held under a quartz plate in a specially constructed 
holder. This optical micrograph taken during the evaporation of a dichloromethane drop placed on the surface reveals the transfer of the TEM grid pattern to the surface-functionalized substrate. Dichloromethane proved the most expedient of several different solvents to photograph the surface pattern. Such patterned substrates were then electrolessly gold-plated, using the three-solution$\mathrm{Sn}(\mathrm{II}) / \mathrm{Ag}(\mathrm{I}) / \mathrm{Au}$ (I) — process beginning with Sn (II) sensitization that had been proven successful for HF-etched $\mathrm{SiN}_{\mathrm{x}}$ (see SI for complete details of metallization solutions and process flow). ${ }^{7,25}$ The pink-tinged, hazy film extending across the substrate in Figure 1c is gold plated onto regions of the $\mathrm{SiN}_{\mathrm{x}}$ that had been subjected to the protection step of photoexposure while immersed in the 1-octene. While gold replicas of the TEM grid masks can be seen in Figure 1c, it is also apparent that the plating spatial selectivity was quite poor compared to its Pd(II)-initiated counterpart, $\mathrm{Pd}(\mathrm{II}) / \mathrm{Ag}(\mathrm{I}) / \mathrm{Au}$ (I) (vide infra, and calculation details in SI). The chemical nature of the surface sensitizing species, and its interactions with the surface, have a strong influence on the performance of a particular electroless plating formulation. The Sn (II) sensitizer is tolerant to substrate composition, which is frequently beneficial, but it is clearly —in this instance, at leastdetrimental to patterned metallization. ${ }^{13,23}$ Figure $1 \mathrm{~d}$ provides a magnified view, by field emission scanning electron microscopy (FE-SEM), of a Sn (II)/Ag (I)/Au (I)-metallized substrate. We did not explore using ultrasonic cleaning steps to improve the plating selectivity, ${ }^{20,26}$ because we wanted to remain compatible with plating free-standing $\mathrm{SiN}_{\mathrm{x}}$ films that are a compelling structural element, especially for nanofluidic devices. ${ }^{3-7}$ 

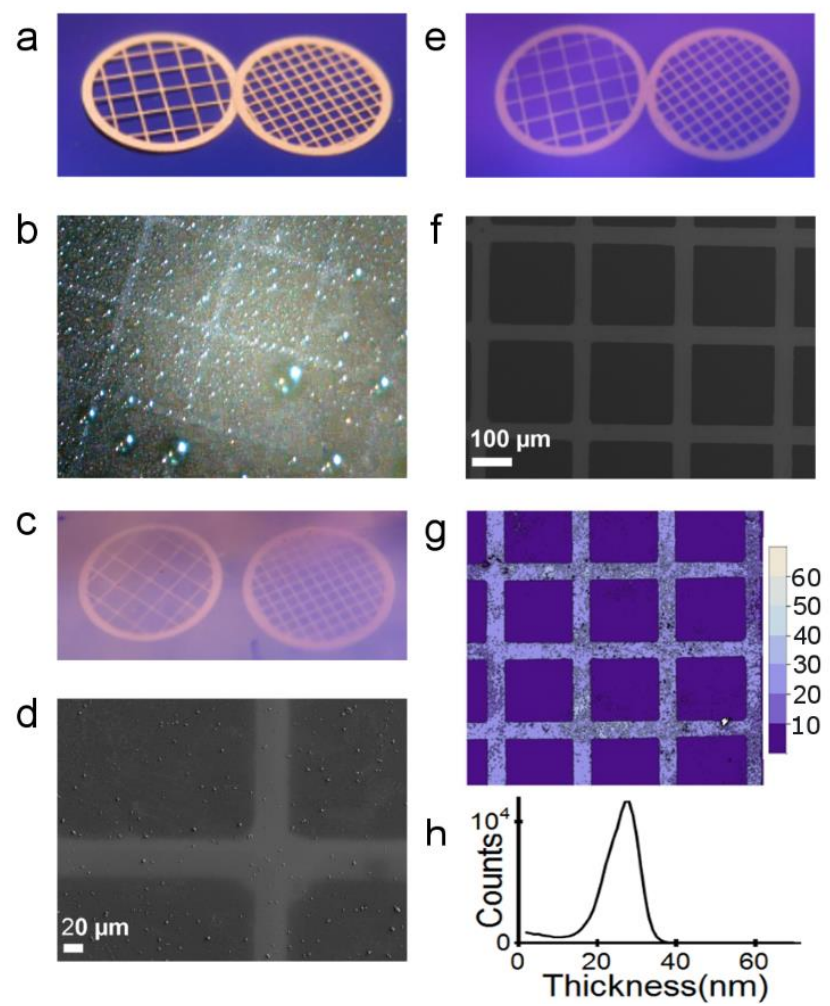

Figure 1: (a) Photographs of 50 and 100 mesh copper TEM grids on a $\mathrm{SiN}_{\mathrm{x}}$-coated silicon chip; (b) micrograph of a 50 mesh 1-octene replica during the evaporation of a dichloromethane drop from a photopatterned chip, with image contrast, gamma, and brightness adjusted for image clarity; (c) photograph of gold replicas after Sn (II) surface sensitization (45 minutes) followed by Ag (I) (5 minutes) and 30 minutes of $\mathrm{Au}(\mathrm{I})$ at $\sim 3^{\circ} \mathrm{C}$, with corresponding (d) FE-SEM image of a 100 mesh pattern; (e) photograph of a gold replica after Pd (II) surface treatment (1 hour) followed $\mathrm{Ag}(\mathrm{I})$ (5 minutes) and 30 minutes of $\mathrm{Au}(\mathrm{I})$ at $\sim 3^{\circ} \mathrm{C}$, with corresponding (f) FE-SEM, (g) DHM (5x magnification) images of a 100 mesh pattern, with color intensity legend denoting film thickness (nm), and (h) histogram giving the film thickness distribution measured inside all of the bars of the micrograph in $(\mathrm{g})$.

We abandoned Sn (II)-sensitized electroless plating when efforts to improve the spatial selectivity by using different rinsing steps, for example, proved ineffective. We tested, instead, a 
palladium-based treatment ${ }^{27}$ in place of the $\mathrm{Sn}$ (II) sensitization step to give an overall process flow of Pd (II)/Ag (I)/Au (I). The use of this Pd (II) surface treatment solution delivered extremely high pattern fidelity, as seen in Figures 1e and 1f. The rich chemistry of the native $\mathrm{SiN}_{\mathrm{x}}$ surface, and of the palladium species, complicates the determination of the mechanism, and indeed may allow for multiple mechanisms to be simultaneously operational. ${ }^{3,13,23,28}$ Figure S2 shows the results of several process chemistry variations, all delivering lower metallized pattern quality than seen in Figures 1e and 1f, but nevertheless highlighting a setting rich with opportunity for fundamental study and applications. For example, substrate photopatterning through an air layerlikely through a photochemical oxidation route similar to that seen on silicon $^{29}$-instead of 1-octene (Figure S2) yielded spatial selectivity degraded by smudges of gold across the surface. The patterned monolayer-templated route offers benefits beyond preserving pattern quality. Photohydrosilylation offers lower process overhead and better compatibility with fluidic channels than conventional photoresist-based approaches, and a suitable hydrosilylated monolayer confers some resistance to any subsequent HF etching, but can be readily removed if necessary (Figure S3). ${ }^{2,18,24}$ The metal plating selectivity when using 1-octene with Pd (II) surface treatment as the first step was easily reproducible across scores of patterned gold depositions when scrupulous adherence to the rinsing steps was maintained. The results shown in Figures 1e and 1f are thus representative and reproducible.

We focus in this work on characterizing the spatial selectivity and the physical structure of the gold layers resulting from this successful initial Pd (II) surface treatment. We present analyses of gold replicas produced after $\sim 30$ minute immersions in the $\mathrm{Au}$ (I) bath. This duration provides a balanced perspective of film nascence and degree of spatial selectivity. Examination of gold replicas using digital holographic microscopy (DHM; Figure 1g) allowed us to determine that the 
gold films were $\sim 23 \pm 1.5 \mathrm{~nm}$ thick. Higher magnification scanning electron micrographs in Figure 2 upheld the quality of selectivity demonstrated in Figures 1e and f. There was only sparse gold coverage where the photoirradiation had installed the protective layer, between the mask grid lines. The gold grid lines, themselves, could be resolved into gold features with $28 \pm 5 \mathrm{~nm}$ mean diameters providing $\sim 83 \%$ surface area coverage (across 15 different grids, with a $13 \%$ standard deviation) after the 30 minutes of immersion in the gold plating bath at $\sim 3^{\circ} \mathrm{C}$. This surface coverage is reflected in measured film (sheet) resistivities of $\sim 10 .{ }_{6} \pm 2.1 \mu \Omega \cdot \mathrm{cm}$ that are unsurprisingly higher than the $2.2 \mu \Omega \cdot \mathrm{cm}$ for bulk gold. ${ }^{7}$ Systematic studies of surface sensitizer preparations have clearly established principles to control the plated film grain size and grain size distribution, and these can be explored for patterning, as well. ${ }^{23}$ The degree of infilling shown here is high in the context of low-process-overhead patterned metallization steps, ${ }^{30}$ and particularly when targeting suitability for use with structured surfaces incompatible with more involved conventional patterning, such as in enclosed micro- and nanofluidic channels.
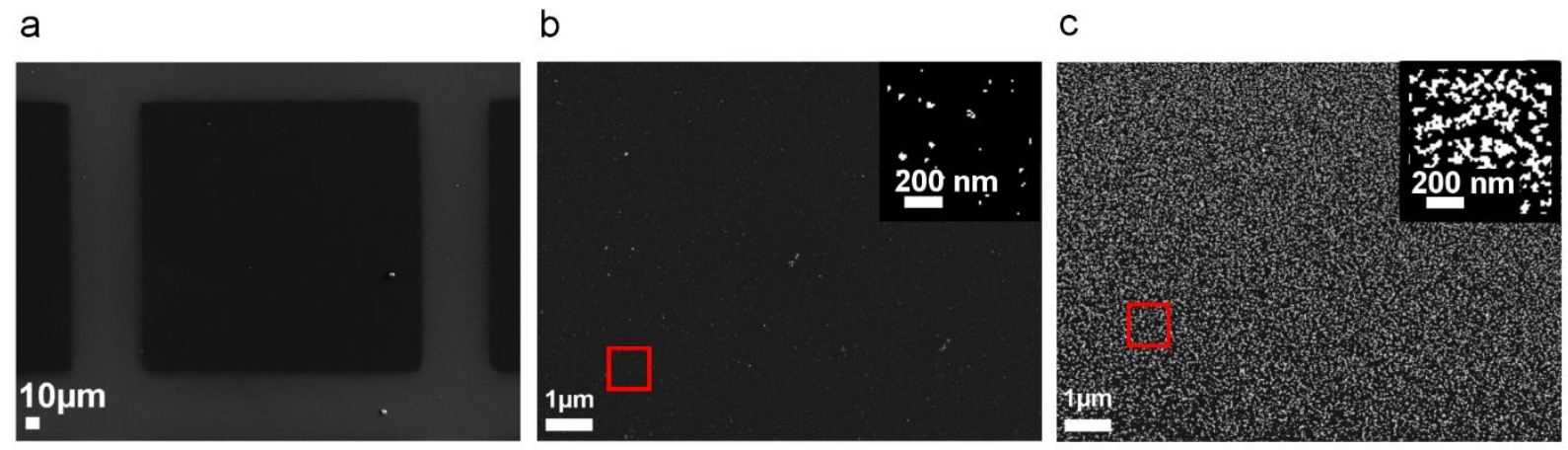

Figure 2: (a) FESEM image of a subsection of a 100 mesh pattern on a $\mathrm{SiN}_{\mathrm{x}}$ chip processed with $\mathrm{Pd}$ (II), $\mathrm{Ag}$ (I), and then Au (I) baths, as detailed in the SI. Vertical and horizontal bars composed of lighter pixels correspond to gold-replicated grid lines on the chip. Zooming into regions outside the bars (b) reveals very little presence of gold grains, confirming the visually observed spatial selectivity as seen in Figure 1d. Zooming into these bars at the same magnification (c) reveals the 
clear grain structure, and high infilling after only 30 minutes of gold plating. The regions of interest bounded by red boxes in (b) and (c) are shown in the inset after the binarization step of the grain size analysis.

To explore the spatial patterning in further detail, we focus on gold replicas of 100 mesh copper grids. The copper bars of these grid masks were $54.4^{ \pm 1.3} \mu \mathrm{m}$ wide (measured by FE-SEM with analysis details in the SI), and they were placed on the $\mathrm{SiN}_{\mathrm{x}}$ surfaces under 1-octene without securing them or attempting to prevent liquid access underneath. The spatial selectivity, defined in a classical signal-to-noise sense (details in the SI), was $\sim 10.1$ for the 1-octene-patterned $\mathrm{Pd}$ (II)/Ag (I)/Au (I) route that we focus on here, in contrast to $\sim 2.7$ for the 1-octene-patterned, Sn (II)-sensitized route, and $\sim 3.2$ for the former solution steps with air-patterning in place of 1-octene. In addition to FE-SEM micrographs, we collected elemental maps from representative gold replicas using energy-dispersive x-ray spectroscopy (EDS; also commonly abbreviated EDX). The maps and electron micrographs in Figure 3a,b are consistent with a thin gold overlayer on $\mathrm{SiN}_{\mathrm{x}}$ that possesses a high degree of infilling and spatial selectivity. We used FE-SEM and EDS line profiles across the open spaces and grid lines to characterize the gold replica lines and the edge resolution, with procedural details provided in the SI. The mean line width of the gold bars in the FE-SEM images of the gold replicas was $44 .{ }_{8} \pm 3.3 \mu \mathrm{m}$, measured from more than 300 lines from each of 9 chips. To extract the edge resolution, we fit the Au-channel EDS intensity versus linear position to Boltzmann functions and recovered sub-micrometer $\left(0.9_{2} \pm 0.2_{4} \mu \mathrm{m} ; 15\right.$ EDS line profiles) transition widths from metal-free to metallized segments. 

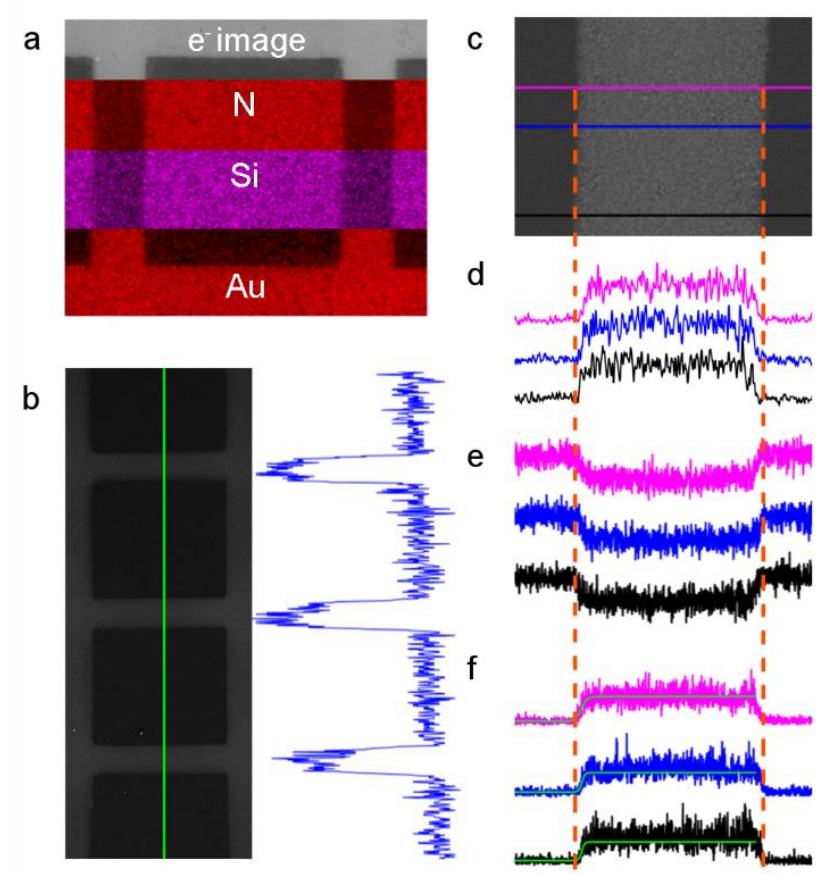

Figure 3: (a) A composite of an electron image (top) and three EDS maps (descending from nitrogen (red) to silicon (purple) to gold (red)). (b) FESEM image of a patterned $\mathrm{SiN}_{\mathrm{x}}$ chip (left) and pixel intensity (right) taken from the micrograph along the green line. (c) Electron image of a subsection of a 100 mesh pattern on a $\mathrm{SiN}_{\mathrm{x}}$ chip. (d) Pixel intensity along each colored line in (c), along with line profiles of spatially-registered EDS maps corresponding to (e) silicon and (f) gold channels (Boltzmann fit is shown in green, with corresponding edge slopes, $d x=0.81,0.59$, and $0.87 \mu \mathrm{m}$ from top to bottom).

We developed a solution-based method to form spatially patterned metal features on silicon-rich $\mathrm{SiN}_{\mathrm{x}}$ thin films. This approach leverages the benefits of electroless plating and establishes a lowoverhead surface-patterning approach suitable for $\mathrm{SiN}_{\mathrm{x}}$ thin films. We ensured that spatial selectivity could be achieved without using ultrasonic excitation or other mechanically disruptive manipulations so that the patterning approach would be compatible with free-standing thin $\mathrm{SiN}_{\mathrm{x}}$ membranes useful in a host of other applications, particularly for micro- and nanofluidics. 
Photochemical hydrosilylation linkage of organic monolayers to $\mathrm{SiN}_{\mathrm{x}}$ is a flexible and appealing route to surface-functionalize $\mathrm{SiN}_{\mathrm{x}}$, especially in conjunction with spatial patterning. The templating monolayer may serve as a permanent or removable coating, protecting the underlying $\mathrm{SiN}_{\mathrm{x}}$ or being removed to expose it after metallization. The ability to readily modify the surface functional groups of these high quality monolayers using standard chemical transformations ${ }^{2}$ dramatically widens the prospects of this simple patterned metallization approach. The alreadyexcellent metallization selectivity could conceivably be further improved and prolonged by tuning the monolayer electrostatics and hydrophobicity, for example. Similarly, the monolayer surface chemistry could be tuned to promote metal layer adhesion if application needs permit the metal layer to rest on the monolayer, itself. ${ }^{9-12,23}$ More tantalizingly, a base monolayer may be used as a platform for further chemical tuning of the surface, in which demonstrated properties and function ${ }^{2}$ can be installed around the patterned gold layer. Thus, we contend that the patterned metallization strategy introduced here is promising and useful not only for delivering a spatially-selective solution-derived metal film, but one primed for further development. ASSOCIATED CONTENT

Supporting Information. Experimental details, method, and sample characterizations. This material is available free of charge via the Internet at http://pubs.acs.org.

\section{AUTHOR INFORMATION}

\section{Corresponding Author}

*E-mail: jdwyer@chm.uri.edu.

\$Present address: Chemistry Department, D64 Hildebrand Hall, UC Berkeley, Berkeley, CA, USA 94720 


\section{Author Contributions}

All authors have given approval to the final version of the manuscript.

\section{Funding Sources}

NSF CAREER award CBET-1150085, in part by NSF EPSCoR Cooperative Agreement \#IIA1330406, and by the University of Rhode Island.

\section{ACKNOWLEDGMENT}

This research has been supported by NSF CAREER award CBET-1150085, in part by NSF EPSCoR Cooperative Agreement \#IIA-1330406 and by the University of Rhode Island, including University of Rhode Island Graduate School Fellowships for Y.M.N.B. (2015) and B.I.K. (2016). We are grateful to Lyncée Tec SA (Lausanne, Switzerland) for graciously providing DHM analyses of our patterned gold films.

\section{ABBREVIATIONS}

SEM, Scanning Electron Microscopy; FE-SEM, Field-Emission SEM; EDS, Energy-Dispersive X-Ray Spectroscopy; LPCVD, Low-Pressure Chemical Vapor Deposition; $\mathrm{SiN}_{\mathrm{x}}$, (Silicon-rich) Silicon Nitride; XPS, X-Ray Photoelectron Spectroscopy; DHM, Digital Holographic Microscopy; RT, Room Temperature.

\section{REFERENCES}

1. Mueller, C.; Harb, M.; Dwyer, J. R.; Miller, R. J. D., Nanofluidic Cells with Controlled Pathlength and Liquid Flow for Rapid, High-Resolution in Situ Imaging with Electrons. J. Phys. Chem. Lett. 2013, 4, 2339-2347. 
2. Arafat, A.; Giesbers, M.; Rosso, M.; Sudhölter, E. J. R.; Schroën, K.; White, R. G.; Yang, L.; Linford, M. R.; Zuilhof, H., Covalent Biofunctionalization of Silicon Nitride Surfaces. Langmuir 2007, 23, 6233-6244.

3. Dwyer, J. R.; Bandara, Y. M. N. D. Y.; Whelan, J. C.; Karawdeniya, B. I.; Nichols, J. W., Silicon Nitride Thin Films for Nanofluidic Device Fabrication. In Nanofluidics, 2nd Edition, 2 ed.; Edel, J.; Ivanov, A.; Kim, M., Eds. Royal Society for Chemistry: 2017; Chapter 7.

4. Haywood, D. G.; Saha-Shah, A.; Baker, L. A.; Jacobson, S. C., Fundamental Studies of Nanofluidics: Nanopores, Nanochannels, and Nanopipets. Anal. Chem. 2015, 87, 172-187.

5. Taniguchi, M., Selective Multidetection Using Nanopores. Anal. Chem. 2015, 87, 188-199.

6. Kudr, J.; Skalickova, S.; Nejdl, L.; Moulick, A.; Ruttkay-Nedecky, B.; Adam, V.; Kizek, R., Fabrication of Solid-State Nanopores and Its Perspectives. ELECTROPHORESIS 2015, 36, 2367-2379.

7. Whelan, J. C.; Karawdeniya, B. I.; Bandara, Y. M. N. D. Y.; Velleco, B. D.; Masterson, C. M.; Dwyer, J. R., Electroless Plating of Thin Gold Films Directly onto Silicon Nitride Thin Films and into Micropores. ACS Appl. Mater. Interfaces 2014, 6, 10952-10957.

8. Dahlin, A. B., Sensing Applications Based on Plasmonic Nanopores: The Hole Story. Analyst 2015, 140, 4748-4759.

9. Jin, Y.; Kang, X.; Song, Y.; Zhang, B.; Cheng, G.; Dong, S., Controlled Nucleation and Growth of Surface-Confined Gold Nanoparticles on a (3-Aminopropyl)TrimethoxysilaneModified Glass Slide: A Strategy for SPR Substrates. Anal. Chem. 2001, 73, 2843-2849. 
10. Asher, T.; Inberg, A.; Glickman, E.; Fishelson, N.; Shacham-Diamand, Y., Formation and Characterization of Low Resistivity Sub-100 nm Copper Films Deposited by Electroless on SAM. Electrochim. Acta 2009, 54, 6053-6057.

11. Flavel, B. S.; Yu, J.; Ellis, A. V.; Quinton, J. S.; Shapter, J. G., Solution Chemistry Approach to Fabricate Vertically Aligned Carbon Nanotubes on Gold Wires: Towards Vertically Integrated Electronics. Nanotechnology 2008, 19, 445301.

12. Vossmeyer, T.; DeIonno, E.; Heath, J. R., Light-Directed Assembly of Nanoparticles. Angew. Chem., Int. Ed. Engl. 1997, 36, 1080-1083.

13. Møller, P.; Nielsen, L. P., Advanced Surface Technology. Møller \& Nielsen APS: Denmark, 2013; Vol. 1, p 594.

14. Menon, V. P.; Martin, C. R., Fabrication and Evaluation of Nanoelectrode Ensembles. Anal. Chem. 1995, 67, 1920-1928.

15. Hulteen, J. C.; Martin, C. R., A General Template-Based Method for the Preparation of Nanomaterials. J. Mater. Chem. 1997, 7, 1075-1087.

16. McCarley, R. L.; Vaidya, B.; Wei, S.; Smith, A. F.; Patel, A. B.; Feng, J.; Murphy, M. C.; Soper, S. A., Resist-Free Patterning of Surface Architectures in Polymer-Based Microanalytical Devices. J. Am. Chem. Soc. 2005, 127, 842-843.

17. Henry, A. C.; McCarley, R. L., Selective Deposition of Metals on Plastics Used in the Construction of Microanalytical Devices: Photo-Directed Formation of Metal Features on PMMA†. J. Phys. Chem. B 2001, 105, 8755-8761. 
18. Carvalho, R. R.; Pujari, S. P.; Lange, S. C.; Sen, R.; Vrouwe, E. X.; Zuilhof, H., Local Light-Induced Modification of the inside of Microfluidic Glass Chips. Langmuir 2016, 32, 23892398.

19. Kwok, H.; Briggs, K.; Tabard-Cossa, V., Nanopore Fabrication by Controlled Dielectric Breakdown. PLoS ONE 2014, 9, e92880.

20. Han, A.; Kuan, A.; Golovchenko, J.; Branton, D., Nanopatterning on Nonplanar and Fragile Substrates with Ice Resists. Nano Lett. 2012, 12, 1018-1021.

21. Santinacci, L.; Djenizian, T.; Hildebrand, H.; Ecoffey, S.; Mokdad, H.; Campanella, T.; Schmuki, P., Selective Palladium Electrochemical Deposition onto AFM-Scratched Silicon Surfaces. Electrochim. Acta 2003, 48, 3123-3130.

22. Guan, F.; Chen, M.; Yang, W.; Wang, J.; Yong, S.; Xue, Q., Fabrication of Patterned Gold Microstructure by Selective Electroless Plating. Appl. Surf. Sci. 2005, 240, 24-27.

23. Zabetakis, D.; Dressick, W. J., Selective Electroless Metallization of Patterned Polymeric Films for Lithography Applications. ACS Appl. Mater. Interfaces 2009, 1, 4-25.

24. Rosso, M.; Giesbers, M.; Arafat, A.; Schroën, K.; Zuilhof, H., Covalently Attached Organic Monolayers on $\mathrm{SiC}$ and $\mathrm{Si}_{\mathrm{x}} \mathrm{N}_{4}$ Surfaces: Formation Using UV Light at Room Temperature. Langmuir 2009, 25, 2172-2180.

25. Whelan, J. C.; Karawdeniya, B. I.; Bandara, Y. M. N. D. Y.; Velleco, B. D.; Masterson, C. M.; Dwyer, J. R., Correction to Electroless Plating of Thin Gold Films Directly onto Silicon Nitride Thin Films and into Micropores. ACS Appl. Mater. Interfaces 2015, 7, 26004-26004. 
26. Kong, Y.; Chen, H.; Wang, Y.; Soper, S. A., Fabrication of a Gold Microelectrode for Amperometric Detection on a Polycarbonate Electrophoresis Chip by Photodirected Electroless Plating. ELECTROPHORESIS 2006, 27, 2940-2950.

27. Ko, J. W.; Koo, H. C.; Kim, D. W.; Seo, S. M.; Kang, T. J.; Kwon, Y.; Yoon, J. L.; Cheon, J. H.; Kim, Y. H.; Kim, J. J.; Park, Y. J., Electroless Gold Plating on Aluminum Patterned Chips for CMOS-Based Sensor Applications. J. Electrochem. Soc. 2010, 157, D46-D49.

28. Porter, L. A.; Choi, H. C.; Ribbe, A. E.; Buriak, J. M., Controlled Electroless Deposition of Noble Metal Nanoparticle Films on Germanium Surfaces. Nano Lett. 2002, 2, 1067-1071.

29. Fabre, B.; Hennous, L.; Ababou-Girard, S.; Meriadec, C., Electroless Patterned Assembly of Metal Nanoparticles on Hydrogen-Terminated Silicon Surfaces for Applications in Photoelectrocatalysis. ACS Appl. Mater. Interfaces 2013, 5, 338-343.

30. Chen, S.-T.; Chen, G.-S., Nanoseeding Via Dual Surface Modification of Alkyl Monolayer for Site-Controlled Electroless Metallization. Langmuir 2011, 27, 12143-12148.

TOC Image

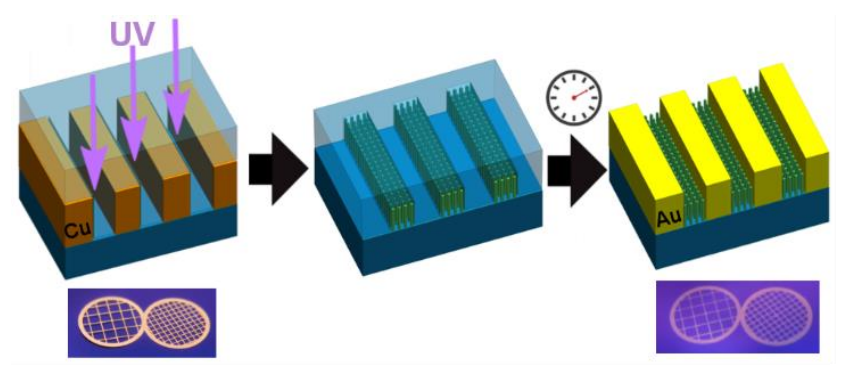

